\title{
Quantum Steering Ellipsoids
}

\author{
Sania Jevtic, ${ }^{1,2}$ Matthew Pusey, ${ }^{3,2}$ David Jennings, ${ }^{2}$ and Terry Rudolph ${ }^{2}$ \\ ${ }^{1}$ Mathematical Sciences, John Crank 501, Brunel University, Uxbridge UB8 3PH, United Kingdom \\ ${ }^{2}$ Controlled Quantum Dynamics Theory, Department of Physics, Imperial College London, \\ London SW7 2AZ, United Kingdom \\ ${ }^{3}$ Perimeter Institute for Theoretical Physics, 31 Caroline Street North, Waterloo, Ontario N2 L 2Y5, Canada
}

(Received 13 May 2013; revised manuscript received 6 December 2013; published 8 July 2014)

The quantum steering ellipsoid of a two-qubit state is the set of Bloch vectors that Bob can collapse Alice's qubit to, considering all possible measurements on his qubit. We provide an elementary construction of the ellipsoid for arbitrary states, calculate its volume, and explain how this geometric representation can be made faithful. The representation provides a range of new results, and uncovers new features, such as the existence of "incomplete steering" in separable states. We show that entanglement can be analyzed in terms of three geometric features of the ellipsoid and prove that a state is separable if and only if it obeys a "nested tetrahedron" condition.

DOI: 10.1103/PhysRevLett.113.020402

PACS numbers: 03.65.Ta, 03.67.Mn

The Bloch sphere provides a simple representation for the state space of the most primitive quantum unit-the qubit-resulting in geometric intuitions that are invaluable in countless fundamental information-processing scenarios. The two-qubit system, likewise, constitutes the primitive unit for bipartite quantum correlations. However, the two-qubit state space is described by 15 real parameters with a surprising amount of structure and complexity. As such, it is challenging both to faithfully represent its states and to acquire natural intuitions for their properties [1-3].

The phenomenon of steering was first uncovered by Schrödinger [4] (and subsequently rediscovered by others [5-7]), who realized that local measurements on Bob's side of the pure state $|\psi\rangle_{A B}$ could be used to "steer" Alice's state into any convex decompositions of her reduced state $\rho_{A}$. Hence, we say that for $|\psi\rangle_{A B}$, steering is "complete" within Alice's Bloch sphere. For a two-qubit mixed state $\rho$, it is known [8] that the convex set of states that Alice can be steered to is an ellipsoid $\mathcal{E}_{A}$, see Fig. 1.

The purpose of this Letter is to show that this steering ellipsoid is the natural generalization of the Bloch sphere picture, in that it can be used to give a faithful representation of an arbitrary two-qubit state in three dimensions, and moreover, that the core properties of the state and its correlations are made manifest in simple geometric terms.

By adopting this representation, we are led to a range of novel results for both separable and entangled states.

First, it reveals a new feature of separable quantum states, called incomplete steering, where not all decompositions of $\rho_{A}$ within the steering ellipsoid $\mathcal{E}_{A}$ are accessible. More

Published by the American Physical Society under the terms of the Creative Commons Attribution 3.0 License. Further distribution of this work must maintain attribution to the author(s) and the published article's title, journal citation, and DOI. importantly, the representation reveals surprising structure in mixed state entanglement. We find that mixed state entanglement decomposes into the simple geometric components of (a) the spatial orientation of the ellipsoid, (b) its distance from the origin, and (c) its size. We are also lead to the surprising nested tetrahedron condition: a state is separable if and only if its ellipsoid fits inside a tetrahedron that itself fits inside the Bloch sphere.

The representation also provides unity and insight for a range of distinct features. The nested tetrahedron condition leads to a simple determination of the minimal number of product states in the ensemble of any separable state. We note that the ellipsoid volume is an entanglement criterion, and provide a formula for it in terms of $\operatorname{det}(\rho)$ and $\operatorname{det}\left(\rho^{T_{B}}\right)$. Nonzero ellipsoid volume is a type of correlation intermediate between discord and entanglement.

Beyond these new insights, we also feel that this method of compactly depicting any two-qubit state in three

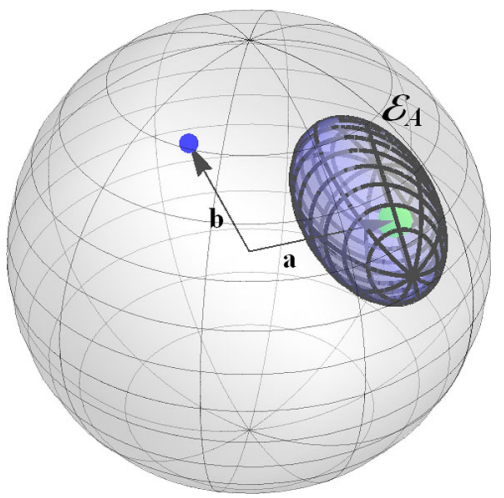

FIG. 1 (color online). Ellipsoid representation of a two-qubit state. For any two-qubit state $\rho$, the set of states to which Bob can steer Alice forms an ellipsoid $\mathcal{E}_{A}$ in Alice's Bloch sphere, containing her Bloch vector $\boldsymbol{a}$. Bob's Bloch vector $\boldsymbol{b}$ is also shown. 
dimensions should be of interest to a range of researchers in both the theoretical and experimental quantum sciences.

The Pauli basis. - Let $\sigma_{\mu}=\left\{\mathbb{1}, \sigma_{x}, \sigma_{y}, \sigma_{z}\right\}, \mu=0,1,2,3$ denote the "homogeneous Pauli basis." Any single-qubit Hermitian operator $\hat{E}$ can be written $\hat{E}=\frac{1}{2} \sum_{\mu=0}^{3} X_{\mu} \sigma_{\mu}$, where the $X_{\mu}=\operatorname{tr}\left(\hat{E} \sigma_{\mu}\right)$ are components of the real vector $X$. Demanding that $\hat{E} \geq 0$ is equivalent to $X_{0} \geq 0$ and $X_{0}^{2} \geq \sum_{i=1}^{3} X_{i}^{2}$, and we can identify $\hat{E}$ as a positive-operator valued measure (POVM) element.

In a similar way, any two-qubit state $\rho$ can be written in the Pauli basis as $\rho=\frac{1}{4} \sum_{\mu, \nu=0}^{3} \Theta_{\mu \nu} \sigma_{\mu} \otimes \sigma_{\nu}$, where $\Theta_{\mu \nu}=\operatorname{tr}\left(\rho \sigma_{\mu} \otimes \sigma_{\nu}\right)$ is real for all $\mu, \nu$. As a block matrix we have $\Theta=\left(\begin{array}{cc}1 & \boldsymbol{b}^{T} \\ \boldsymbol{a} & T\end{array}\right)$, where $\boldsymbol{a}, \boldsymbol{b}$ are the Bloch vectors of the reduced states $\rho_{A}$ and $\rho_{B}$ of $\rho$, respectively, and $T$ is a $3 \times 3$ matrix encoding the correlations [2]. If Bob does a POVM and obtains outcome $\hat{E}$, he steers Alice to the state proportional to $\operatorname{tr}_{B}[\rho(\mathbb{1} \otimes \hat{E})]$, which, in the Pauli basis, is given by the four-vector $\frac{1}{2} \Theta X$, with probability $\frac{1}{2}(1+\boldsymbol{b} \cdot \boldsymbol{x})$ where $\boldsymbol{x}=\left(X_{1}, X_{2}, X_{3}\right)^{T}$.

The four-vector formalism is related to the idea of stochastic local operations and classical communication (SLOCC) [1], which are operations of the form $\rho \rightarrow \rho^{\prime}=S_{A} \otimes S_{B} \rho\left(S_{A} \otimes S_{B}\right)^{\dagger}$, where $S_{A}, S_{B}$ are invertible complex matrices. The set of states attainable from $\rho$ under SLOCC is called the SLOCC orbit of $\rho$, and denoted $\mathcal{S}(\rho)$. Under this action, the matrix $\Theta$ transforms as $\Theta^{\prime}=$ $\Lambda_{A} \Theta \Lambda_{B}^{T}$ where $\Lambda_{A(B)}$ are proper orthochronous Lorentz transformations [9]. Significant in what follows, for a SLOCC operation affecting only Bob $\left(\Theta^{\prime}=\Theta \Lambda_{B}\right)$, the set of states Alice is steered to is unaffected, since: $X$ is in the forward light cone if and only if $X^{\prime}=\Lambda_{B} X$ is, and $\Theta^{\prime} X=\Theta X^{\prime}$.

Previously, in [8], a range of SLOCC techniques were employed to study entanglement and steering for two-qubit mixed states; however, this approach encounters problems when applied to certain separable states and, moreover, is not suited to addressing the geometric features of interest. The techniques developed here follow a different line, and circumvent both of these issues.

Construction of the quantum steering ellipsoid.-We now provide an alternative construction of the steering ellipsoid $\mathcal{E}_{A}$ to that in [15], which applies even when $\mathcal{E}_{A}$ is degenerate.

Our construction of $\mathcal{E}_{A}$ is easiest to understand in the case when the state $\rho$ has $\boldsymbol{b}=\mathbf{0}$. For such a state, suppose Bob projects his qubit onto the pure state $X=\left(\begin{array}{l}1 \\ \boldsymbol{x}\end{array}\right)$ with $x=1$. Given this outcome, Alice is steered to

$$
Y=\Theta X=\left(\begin{array}{cc}
1 & \mathbf{0}^{T} \\
\boldsymbol{a} & T
\end{array}\right)\left(\begin{array}{c}
1 \\
\boldsymbol{x}
\end{array}\right)=\left(\begin{array}{c}
1 \\
\boldsymbol{a}+T \boldsymbol{x}
\end{array}\right)
$$

which occurs with probability $\frac{1}{2}$, and where Alice's Bloch vector is now $\boldsymbol{a}+T \boldsymbol{x}$. The set of all states Alice can end up with is simply the unit sphere of possible $\boldsymbol{x}$, shrunk and rotated by $T$ and translated by $\boldsymbol{a}$, i.e., an ellipsoid centered at $\boldsymbol{a}$ with orientation and semiaxes given by the eigenvectors and eigenvalues of $T T^{T}$. The ellipsoid dimension is $\operatorname{rank}(T)=\operatorname{rank}(\Theta)-1$. Points inside the ellipsoid can be reached via convex combinations of projective measurements, and conversely, a POVM element is a positive operator and so can be spectrally decomposed into a mixture of projectors, thus, giving a point within the ellipsoid.

Now, consider a general state with $\boldsymbol{b} \neq \mathbf{0}$. If $b=1$, then $\rho$ is a product state, in which case there is no steering and the steering ellipsoid is the single point $\boldsymbol{a}$. For the case $b<1$, we find that the SLOCC operator $\mathbb{1} \otimes\left(2 \rho_{B}\right)^{-(1 / 2)}$ corresponds to a Lorentz boost $L_{\boldsymbol{b}}$ by a "velocity" $\boldsymbol{b}$ that transforms $\rho_{B}$ to the maximally mixed state (which has $\boldsymbol{b}=\mathbf{0})$. We refer to this special filtered state $\tilde{\rho}$ as the "canonical state" on the SLOCC orbit $\mathcal{S}(\rho)$. Since SLOCC operations on Bob do not affect Alice's steering ellipsoid, the parameters of an arbitrary state's steering ellipsoid are obtained by simply boosting $\Theta$ by $L_{\boldsymbol{b}}$ and reading off the ellipsoid parameters. This gives a steering ellipsoid centered at $\boldsymbol{c}_{A}=(\boldsymbol{a}-T \boldsymbol{b}) /\left(1-b^{2}\right)$, with orientation and semiaxes lengths $s_{i}=\sqrt{q_{i}}$ given [9] by the eigenvectors and eigenvalues $q_{i}$ of the ellipsoid matrix

$$
Q_{A}=\frac{1}{1-b^{2}}\left(T-\boldsymbol{a} \boldsymbol{b}^{T}\right)\left(\mathbb{1}+\frac{\boldsymbol{b} \boldsymbol{b}^{T}}{1-b^{2}}\right)\left(T^{T}-\boldsymbol{b} \boldsymbol{a}^{T}\right) .
$$

To obtain $\mathcal{E}_{B}$, the ellipsoid at $\mathrm{B}$, we simply perform a swap of $A$ and $B$, which corresponds to transposing $\Theta$ and sends $\boldsymbol{b} \rightarrow \boldsymbol{a}, \boldsymbol{a} \rightarrow \boldsymbol{b}, T \rightarrow T^{T}$. Hence, $\mathcal{E}_{A}$ and $\mathcal{E}_{B}$ always have the same dimensionality, $\operatorname{rank}(\Theta)-1$. This completes the construction of the geometric data $\left(\mathcal{E}_{A}, \boldsymbol{a}, \boldsymbol{b}\right)$ for a given state $\rho$. Next, we describe the reverse direction: obtaining $\rho$ from an ellipsoid $\mathcal{E}_{A}$ and the vectors $\boldsymbol{a}$ and $\boldsymbol{b}$.

Reconstruction of $\rho$ from geometric data.-Given $\boldsymbol{a}, \boldsymbol{b}, \mathcal{E}_{A}=\left(Q_{A}, \boldsymbol{c}_{A}\right)$, to recover $\rho$, we need $T$. In [9], we prove that this matrix is given by

$$
T=\frac{1}{\gamma}\left(\gamma \boldsymbol{c}_{A} \boldsymbol{b}^{T}+\sqrt{Q_{A}} O+\frac{\gamma-1}{b^{2}} \sqrt{Q_{A}} O \boldsymbol{b} \boldsymbol{b}^{T}\right),
$$

where $O \in \mathrm{O}(3)$ satisfies $\boldsymbol{a}=\boldsymbol{c}_{A}+\sqrt{Q_{A}} O \boldsymbol{b}$. This specifies $O$ up to a rotation $O^{\prime} \in \mathrm{O}(3)$ such that $O^{\prime} \boldsymbol{b}=\boldsymbol{b}$. The action of $O^{\prime}$ can be encoded, for example, by a coloring of $\mathcal{E}_{A}$ as in [16]. In this way, the steering ellipsoid can be used as a faithful representation of $\rho$. $O^{\prime}$ corresponds to a local unitary and/or partial transpose on Bob's system, and so is irrelevant for any correlation properties such as entanglement.

"Complete" and "incomplete" steering.-The steering ellipsoid specifies which states Bob can steer Alice to. A more subtle question is which decompositions of 
Alice's reduced state he can steer to. Clearly, a necessary condition is that all of the states in the decomposition must be in $\mathcal{E}_{A}$, surprisingly however, it turns out that this is not sufficient.

Consider some nonproduct two-qubit state with ellipsoids $\mathcal{E}_{A}$ and $\mathcal{E}_{B}$. The following are equivalent [9]: (1) (Complete steering of A) For any convex decomposition of $\boldsymbol{a}$ into vectors in $\mathcal{E}_{A}$ or on its surface, there exists a POVM for Bob that steers Alice to it. (2) The affine span of $\mathcal{E}_{B}$ contains the origin.

These conditions hold for all nondegenerate ellipsoids (which includes all entangled states) as well as all states where $\boldsymbol{b}=\mathbf{0}$. However, complete steering is not symmetric: the state $\rho=\frac{1}{2}(|00\rangle\langle 00|+|+1\rangle\langle+1|)$ has complete steering of Alice by Bob, but not vice versa.

The three geometric contributions to entanglement.The Peres-Horodecki criterion $[17,18]$ asserts that a twoqubit state $\rho_{e}$ is entangled if and only if $\rho_{e}^{T_{B}}$ has a negative eigenvalue. Furthermore, it can be shown [15] that, at most, one eigenvalue of $\rho_{e}^{T_{B}}$ can be negative, and that $\rho_{e}^{T_{B}}$ is full rank for all entangled states [19]. Hence, $\operatorname{det} \rho_{e}^{T_{B}}<0$ is a necessary and sufficient condition for entanglement.

Suppose $\rho$ is entangled, then any state in its SLOCC orbit $\mathcal{S}(\rho)$ is also entangled [15], including the canonical state $\tilde{\rho} \in \mathcal{S}(\rho)$. It follows that $\rho$ is entangled if and only if $\operatorname{det}\left(\tilde{\rho}^{T_{B}}\right)<0$. However, the states $\rho$ and $\tilde{\rho}$ share the same $\mathcal{E}_{A}$, and so, expanding $\operatorname{det}\left(\tilde{\rho}^{T_{B}}\right)<0$ in the geometric representation, we find that $\rho$ is entangled if and only if a physical steering ellipsoid with center $\boldsymbol{c}=c \hat{\boldsymbol{n}}$ and matrix $Q$ satisfies

$$
c^{4}-2 c^{2}\left(1-\operatorname{tr} Q+2 \hat{\boldsymbol{n}}^{T} Q \hat{\boldsymbol{n}}\right)+h(Q)<0,
$$

where $h(Q):=1-8 \sqrt{\operatorname{det} Q}+2 \operatorname{tr}\left(Q^{2}\right)-[\operatorname{tr}(Q)]^{2}-2 \operatorname{tr}(Q)$, and we drop $A, B$ labels as entanglement is a "symmetric" relation. This equation is manifestly invariant under global rotations, corresponding to local unitaries on the quantum state, and shows that correlations between the qubits manifest themselves in three geometric ways: (1) the distance $c$ of the ellipsoid center from the origin, (2) the size of the ellipsoid, and (3) its "skew," captured by the term $\hat{\boldsymbol{n}}^{T} Q \hat{\boldsymbol{n}}$, which reflects the alignment of the ellipsoid relative to the radial direction described by center unit vector $\hat{\boldsymbol{n}}$.

The nested tetrahedron condition.-The condition for entanglement given by equation (4) provides a compact algebraic condition for nonseparability and uncovers contributions from different geometric aspects. However, the steering ellipsoid captures the distinction between separable and nonseparable states in another elegant way: A two-qubit state $\rho$ is separable if and only if its steering ellipsoid $\mathcal{E}_{A}$ fits inside a tetrahedron that fits inside the Bloch sphere. To prove necessity, suppose Alice and Bob share a separable state $\rho=\sum_{i=1}^{n} p_{i} \alpha_{i} \otimes \beta_{i}$. Since we can always take $n \leq 4$ [20], the Bloch vectors of the $\alpha_{i}$ define a (possibly degenerate) tetrahedron $\mathcal{T}$ within Alice's Bloch sphere. Bob's outcome $\hat{E}$ collapses Alice to $\sum_{i=1}^{n}\left(\operatorname{tr}\left(\hat{E} \beta_{i}\right) / \operatorname{tr}\left(\hat{E} \rho_{B}\right)\right) p_{i} \alpha_{i}$. Hence, her steered Bloch vector will be a convex combination of the Bloch vectors for the $\alpha_{i}$-in other words her steering ellipsoid is contained in $\mathcal{T}$.

We prove, in [9], that the nontrivial converse holds: any ellipsoid that fits inside a tetrahedron that itself fits inside the Bloch sphere must arise from a separable state, and thus, the nested tetrahedron condition is both necessary and sufficient for separability of the state.

This key geometric insight leads to some nontrivial corollaries. For example, for any separable state $\rho$, the minimal number of product states in an ensemble decomposition $\rho=\sum_{i=1}^{n} p_{i} \alpha_{i} \otimes \beta_{i}$ is $n=\operatorname{rank}(\Theta)$. If $\operatorname{rank}(\Theta)=1$, we have a product state, and so $n=1$, while if $\operatorname{rank}(\Theta)=2$, we have that $\mathcal{E}_{A}$ is a line segment and we form a decomposition of $\rho$ using the endpoints of this segment, giving $n=2$. The case $\operatorname{rank}(\Theta)=3$ is slightly more involved, but follows from the fact any ellipse inside a tetrahedron inside the unit sphere also lies inside a triangle in the unit sphere $[9,21]$. Finally, it is known [20] that any separable state can be written using four product states, which covers the case $\operatorname{rank}(\Theta)=4$. Combining this with the above results on complete steering provides a natural geometrical classification of two qubit states, as in Fig. 2.

Quantum discord and ellipsoid orientation.-Quantum discord has received much attention as a measure of the quantumness of correlations (see [22] for details) in which zero discord for one party roughly corresponds to them possessing a nondisturbing projective measurement. Within the geometric representation, it is readily seen that $\rho$ has zero discord for $A$ if and only if $\mathcal{E}_{A}$ degenerates to a radial line segment, while $\rho$ has zero discord for $B$ if and only if $\mathcal{E}_{A}$ is one dimensional and $b=2\left|\boldsymbol{c}_{A}-\boldsymbol{a}\right| / l_{A}$, where $l_{A}$ is the length of $\mathcal{E}_{A}$ [9].

To illustrate the effect of the alignment of $\mathcal{E}_{A}$ on the entanglement and discord of a state, we can consider a one-parameter family of states of the form $\rho(\theta)=\frac{1}{4}(\mathbb{1}+$ $\left.\frac{1}{2} \sigma_{z} \otimes \mathbb{1}+\sum_{i j} T_{i j}(\theta) \sigma_{i} \otimes \sigma_{j}\right)$, for which the ellipsoid skew varies smoothly with $\theta$ while maintaining a constant volume for $\mathcal{E}_{A}$. Specifically, we have that $T(\theta)=$ $R_{y}(\theta) K R_{y}^{T}(\theta)=\sqrt{Q_{A}(\theta)}$, and so $R_{y}(\theta) \in \mathrm{SO}(3)$ generates states with inequivalent correlations via rotation of the steering ellipsoid around its own center $\boldsymbol{c}_{A}=\left(0,0, \frac{1}{2}\right)^{T}$, note that this "internal rotation" is distinct from a global rotation generated by a local unitary on the state. We choose $K=\operatorname{diag}(-(9 / 20),-(3 / 10),-(3 / 10))$ so that $\rho(\theta) \geq 0$, for all $\theta \in[0, \pi)$. This family of states illustrates opposing behavior of the discord and concurrence as a function of $\theta$, see Fig. 3. The entanglement favors an orientation in which the longest semiaxis is aligned (radial) with $c_{A}$ at the point $\theta=\pi / 2$, while discord is maximized when the short semiaxis is radial, at $\theta=0, \pi$ [23].

Volume of the ellipsoid.-The expression for the volume of $\mathcal{E}_{A}$ provides a compact and nontrivial relation between 


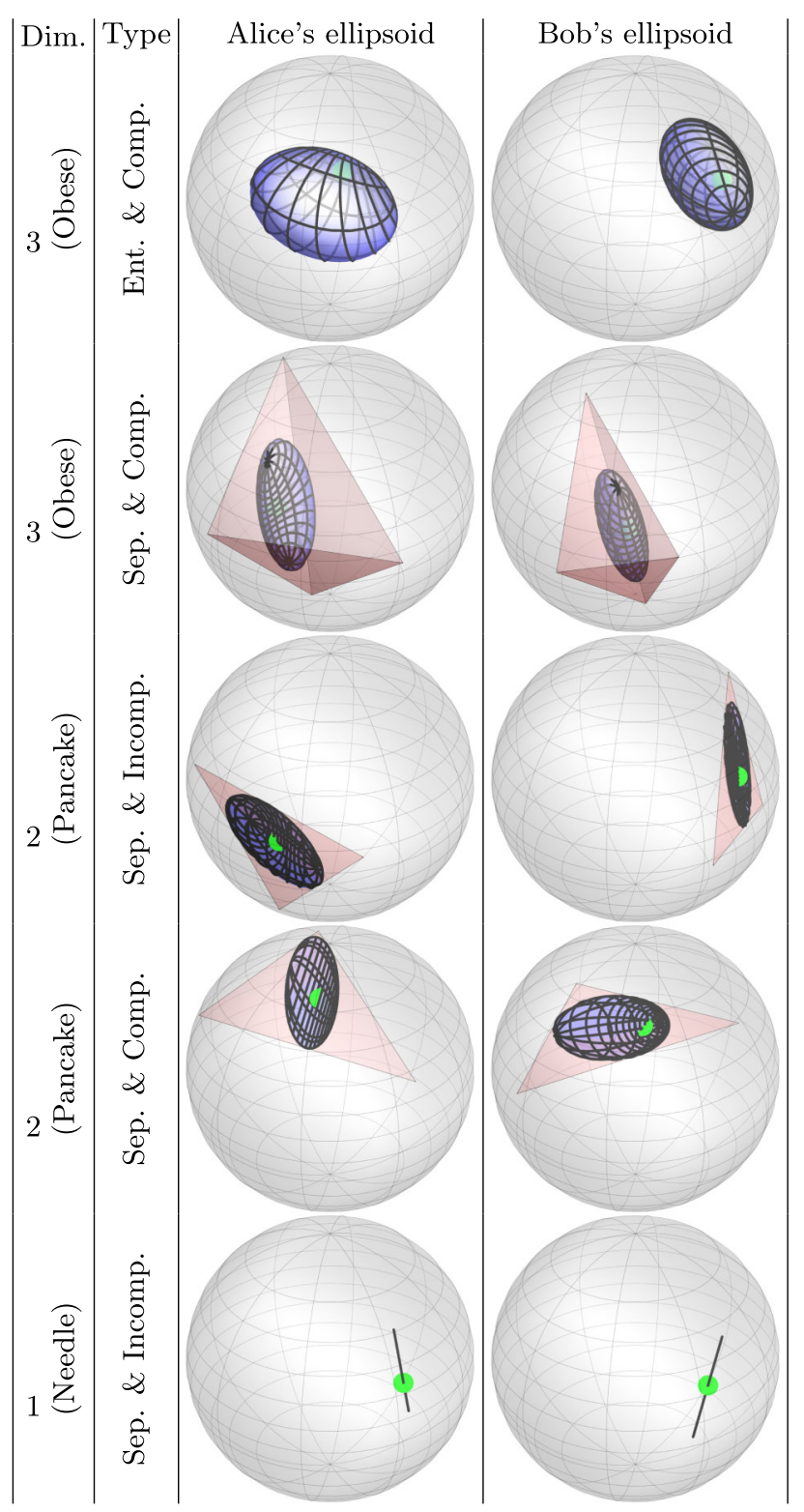

FIG. 2 (color online). The classes of steering ellipsoids. Here, "Separable" (Sep.) and "Entangled" (Ent.) label the type of correlation, while "Incomplete" (Incomp.) and "Complete" (Comp.) label the type of steering. The large (green) dot is the reduced state in the respective Bloch sphere. States with $\mathcal{E}_{A}$, being three dimensional, have nonzero volume (or simply "obesity"), and these are either entangled or separable. The state $\rho$ is separable if and only if $\mathcal{E}_{A}$ fits inside a tetrahedron inside the Bloch sphere of $A$. For separable states, the set $\mathcal{E}_{A}$ can also be two dimensional (a steering pancake), or one dimensional (a steering needle), or trivially zero dimensional (not shown). For these cases, steering is either "complete," if all ensemble decompositions of $\boldsymbol{a}$ in $\mathcal{E}_{A}$ are attainable (when the span of $\mathcal{E}_{B}$ contains $\frac{1}{2} \mathbb{1}$ ), otherwise, the steering is "incomplete." Zero discord occurs only for radial steering needles.

the steering properties of $\rho$ and the ranks of $\rho$ and $\rho^{T_{B}}$. The volume of any ellipsoid is proportional to the product of its semiaxes $V=(4 \pi / 3) s_{1} s_{2} s_{3}$. Therefore, $\mathcal{E}_{A}$ has volume $V_{A}=(4 \pi / 3) \sqrt{\operatorname{det} Q_{A}}$. Using the ellipsoid

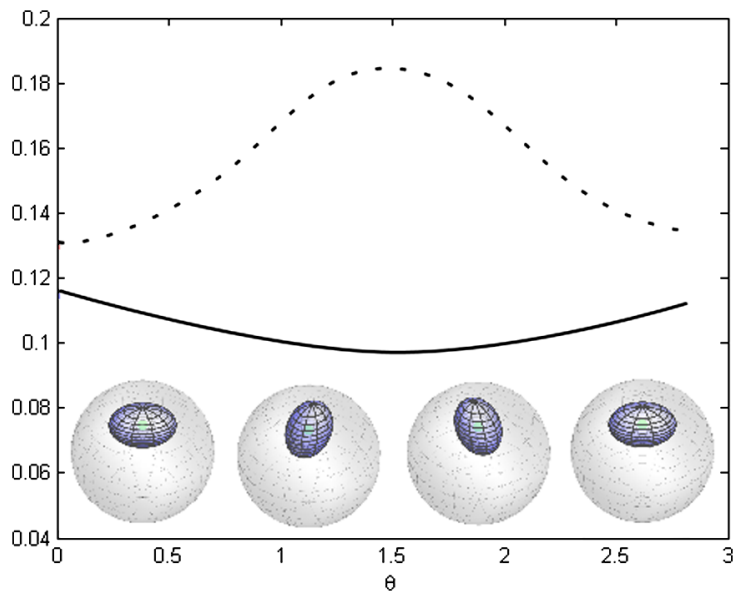

FIG. 3 (color online). Discord (solid curve) and concurrence (dotted curve) of the state $\rho(\theta)$ as a function of the orientation $\theta$ of the ellipsoid. Entanglement is maximized when the major axis is radial.

matrix in equation (2), which may be rewritten as $V_{A}=(4 \pi / 3)\left(|\operatorname{det} \Theta| /\left(1-b^{2}\right)^{2}\right)$. However, it turns out [9] that $\operatorname{det} \Theta=16\left(\operatorname{det} \rho^{T_{B}}-\operatorname{det} \rho\right)$; therefore,

$$
V_{A}=\frac{64 \pi}{3} \frac{\left|\operatorname{det} \rho-\operatorname{det} \rho^{T_{B}}\right|}{\left(1-b^{2}\right)^{2}} .
$$

The $\mathcal{E}_{B}$ volume follows from $V_{A}$ via the simple relation $V_{B}=V_{A}\left(1-b^{2}\right)^{2} /\left(1-a^{2}\right)^{2}$.

The ellipsoid volume is a nonlinear entanglement criterion. Specifically, the Werner state on the separableentangled boundary has $\mathcal{E}_{A}$ being the maximal sphere volume $V_{\star}=4 \pi / 81$ inscribed inside the largest possible tetrahedron that can be inscribed inside the unit sphere [25]. We immediately deduce that any state with $\mathcal{E}_{A}$ that has volume $V>V_{\star}$ must be entangled. Note that entangled states can have $V \leq V_{\star}$.

Since $V>V_{\star}$ can only be attained by entangled states, whilst zero discord states have one-dimensional (degenerate) ellipsoids, we see that nonzero volume, or "obesity," is strictly stronger than discord but strictly weaker than entanglement.

Conclusion.-The quantum steering ellipsoid provides a faithful representation of any two-qubit state and a natural geometric classification of states. It yields clear and intuitive understanding into the usual key aspects of two-qubit states, uncovers surprising new features (such as the nested tetrahedron condition, skew and obesity, and incomplete steering) while prompting novel questions, such as: can we use (4) to define a class of "least-classical" separable states for fixed $(\boldsymbol{a}, \boldsymbol{b}, \boldsymbol{c})$ ? Can we use the nested tetrahedron condition to provide a simple construction for the best separable approximation [28] for a state $\rho$ ? What is the geometric characterization of the possible steering ellipsoids for an arbitrary two-qubit state? This would 
potentially be useful when using ellipsoids to visualize the results of two-qubit state tomography. In [9], we have provided a discussion of several extensions to the work described in this Letter, beyond the two-qubit scenario to higher dimensional systems.

We wish to acknowledge Antony Milne for his useful comments, and Zuzana Gavorova and Peter Lewis for their early contributions to this topic. M. P. and S. J. acknowledges support by the EPSRC; S. J. acknowledges funding by EPSRC Grant No. EP/K022512/1; D. J. acknowledges funding by the Royal Commission for the Exhibition of 1851; T.R. acknowledges support by the Leverhulme Trust. Research at Perimeter Institute is supported in part by the Government of Canada through NSERC and by the Province of Ontario through MRI.

[1] J. E. Avron, G. Bisker, and O. Kenneth, J. Math. Phys. (N.Y.) 48, 102107 (2007).

[2] R. Horodecki and M. Horodecki, Phys. Rev. A 54, 1838 (1996).

[3] I. Bengtsson and K. Życzkowski, Geometry of Quantum States: An Introduction to Quantum Entanglement (Cambridge University Press, Cambridge, England, 2006).

[4] E. Schrödinger, Proc. Cambridge Philos. Soc. 31, 555 (1935).

[5] N. Gisin, Phys. Lett. A 210, 151 (1996).

[6] L. P. Hughston, R. Jozsa, and W. K. Wootters, Phys. Lett. A 183, 14 (1993).

[7] R. W. Spekkens and T. Rudolph, Quantum Inf. Comput. 2, 66 (2001).

[8] F. Verstraete, Ph.D. thesis, Katholieke Universiteit Leuven (2002).

[9] See Supplemental Material at http://link.aps.org/ supplemental/10.1103/PhysRevLett.113.020402 which includes Refs. [10-14], for details.

[10] R. Frosch, Four-tensors, the Mother Tongue of Classical Physics (VDF Hochschulverlag, Zurich, 2006).

[11] B. Dakić, V. Vedral, and Č. Brukner, Phys. Rev. Lett. 105, 190502 (2010).

[12] S. K. Goyal, B. Neethi Simon, R. Singh, and S. Simon, arXiv:1111.4427.
[13] L. Chen and D. Ž. Đoković, Phys. Rev. A 86, 062332 (2012).

[14] A. Milne, S. Jevtic, D. Jennings, H. Wiseman, and T. Rudolph, arXiv:1403.0418.

[15] F. Verstraete, J. Dehaene, and B. DeMoor, Phys. Rev. A 64, 010101 (2001).

[16] J. B. Altepeter, E. R. Jeffrey, M. Medic, and P. Kumar, in Conference on Lasers and Electro-Optics, 2009 and 2009 Quantum Electronics and Laser Science Conference [i.e. International Quantum Electronics Conference]: CLEO/ QELS [i.e. CLEO/IQEC] 2009; June 2009, Baltimore, MD, USA; [including] PhAST 2009 (IEEE/Optical Society of America, Piscataway, NJ, 2009), p. IWC1.

[17] A. Peres, Phys. Rev. Lett. 77, 1413 (1996).

[18] M. Horodecki, P. Horodecki, and R. Horodecki, Phys. Lett. A 223, 1 (1996).

[19] A. Sanpera, R. Tarrach, and G. Vidal, Phys. Rev. A 58, 826 (1998).

[20] W. K. Wootters, Phys. Rev. Lett. 80, 2245 (1998).

[21] M. Vidrighin, "mathoverflow.net/questions/109153," (2012).

[22] H. Ollivier and W. H. Zurek, Phys. Rev. Lett. 88, 017901 (2001).

[23] Discord can be calculated analytically when $\theta=0, \pi / 2$, and at these points $\rho$ is an X state [24] and this agrees with our findings.

[24] C. S. Mingjun Shi, Fengjian Jiang, and J. Du, New J. Phys. 13, 073016 (2011).

[25] More formally, it was shown [26,27] that every convex body in $\mathbb{R}^{n}$ Euclidean space contains a unique maximal volume ellipsoid, and that a Euclidean ball is that maximum if and only if there exist vectors $\left\{\boldsymbol{x}_{k}\right\}$ in the set of contact points of the ball with the convex body, and positive numbers $\left\{c_{k}\right\}$ such that $\sum_{k} c_{k} \boldsymbol{x}_{k}=0$ and $\sum_{k} c_{k} \boldsymbol{x}_{k}^{T} \boldsymbol{x}_{k}=r^{2} \mathbb{1}$, where $r$ is the radius of the ball. Inspection of the insphere for the regular tetrahedron in the Bloch sphere shows that it is the case for $\left\{\boldsymbol{x}_{k}\right\}$ being the four intersection points.

[26] F. John, in Studies and Essays Presented to R. Courant on his 60th Birthday, Jan. 8, 1948 (Interscience, New York, 1948), pp. 187-204.

[27] K. Ball, Geometriae Dedicata 41, 241 (1992).

[28] M. Lewenstein and A. Sanpera, Phys. Rev. Lett. 80, 2261 (1998). 